\title{
The Effect of Vitamin C Addition on Epigallocatechin Gallate (EGCG) Stability in Green Tea Solution
}

\author{
Alief Putriana Rahman ${ }^{1}$, Djoko Agus Purwanto ${ }^{2}$, Isnaeni ${ }^{2}$ * \\ ${ }^{1}$ Master of Pharmaceutical Science, Faculty of Pharmacy, Universitas Airlangga, Surabaya \\ ${ }^{2}$ Departement of Pharmaceutical Chemistry, Faculty of Pharmacy, Universitas Airlangga, Surabaya \\ *Corresponding author: isna.yudi@gmail.com
}

Submitted: 5 Desember 2019

Accepted: 15 Januari 2020

Published: 29 Februari 2020

\begin{abstract}
Background: Epigallocatechin gallate (EGCG) is the most abundant green tea catechin with a powerful antioxidant effect to prevent cancer cells. EGCG in green tea solution is highly susceptible to degradation, this it is urgent to increase the stability of EGCG by vitamin C addition. Vitamin $C$ can regenerate radical EGCG to be normal EGCG. Objective: This research aim was to determine percent of decreased level of EGCG before and after vitamin $C$ addition. Methods: This paper was focused on enhancing the stability of EGCG by $1 \mathrm{mg}$ (GTVC1), $1.5 \mathrm{mg}$ (GTVC2), $2 \mathrm{mg}$ (GTVC3), $2.5 \mathrm{mg}$ (GTVC4) and $3 \mathrm{mg}$ (GTVC5) of vitamin C addition to $10 \mathrm{~g} / \mathrm{L}$ of green tea solution concentration. Evaluations of EGCG were conducted at 0 days, 1 day, 2 days, 3 days, and 4 days of storage time. EGCG was analyzed using thin layer chromatography (TLC) densitometry methods. The stability of EGCG was determined by \% of decreased EGCG. Results: Percent of loss of EGCG in GT, GTVCl, GTVC2, GTVC3, GTVC4 and GTVC5 after 4 days storage were 19.93\%, 10.89\%, 21.08\%, 18.18\%, 28.56\%, and 9.76\%, respectively. Conclusion: This study showed that in 4 days storage, the decreased level of EGCG in green tea solution with $3 \mathrm{mg}$ of vitamin C addition (GTVC5) was $9.76 \%$ which was smaller than green tea solution without vitamin C addition (GT) which EGCG decreasing $19.93 \%$.
\end{abstract}

Keywords: EGCG, vitamin C, stability, green tea, TLC

\section{INTRODUCTION}

Tea is the most widely consumed beverage in the world and derived from leaves of the plant Camellia sinensis. In Indonesia, tea is one of the agricultural main products and commodity exports. Green tea is a type of tea that has the greatest health benefit owing to high radical scavenging ability. Indonesia recognized as the third-largest green tea consumption country in Asia. It was known that about 30,000 ton green tea was consumed annually (Kosugi, 2019).

Green tea contains a number of biologically active compounds, which include (-)-epicatechin (EC), (-)epicatechin-3-gallate (ECG), (-)-epigallocatechin (EGC), and (-)-epigallocatechin-3 gallate (EGCG) (Rady et al., 2018).

Around $59 \%$ of the total catechin from the leaves of the green tea is (-) epigallocatechin-3 gallate (EGCG) (Rady et al., 2018). The radical scavenging ability of EGCG was higher than other catechins because EGCG has the number of hydroxyl groups much more than others. That is why, EGCG can inhibit or reduce cancer cells in the human body (Wu et al., 2011). Nevertheless, the chemical structure of EGCG makes it susceptibles to degradation. It is caused by auto-oxidation and epimerization process. The autooxidation degrades EGCG to be theasinensin A and epimerization degrade EGCG to be GCG or gallocatechin gallate (Krupkova et al., 2016).

The degradation of EGCG is affected by parameters such as extraction of brewing methods, storage temperature, and type of storage bottle. For this research, all of the parameters were used in optimum condition from the previous study. Ultrasonic extraction was used for brewing the sample at temperature $80^{\circ} \mathrm{C}$ (Das \& Eun, 2018). Dark bottle was used for storage to avoid the light (Zeng et al., 2017). The temperature of storage was set at $4^{\circ} \mathrm{C}$ (Sigma \& Zeng et al., 2017).

The previous study explained that vitamin $\mathrm{C}$ or Ascorbic acid addition could stabilize the content of 
EGCG in green tea brewing. Vitamin $\mathrm{C}$ was found to regenerate EGCG radicals. The interactions between EGCG and vitamin $\mathrm{C}$ could be synergitic. The synergistic effect wass considered to occur owing to the difference of the reduction potential (Dai et al., 2008). The reduction potential of EGCG was $430 \mathrm{mV}$ and the reduction potential of vitamin C was $282 \mathrm{mV}$ (Dai et al., 2008). Based on that, vitamin C was easier to transfer electron and EGCG was also easier to attract electron. EGCG degradation would attract electrons from vitamin $\mathrm{C}$ easily, so EGCG radical regenerated to normal EGCG. Oxidation of vitamin $\mathrm{C}$ forms oxalic and trienoic acid (Truffault et al., 2017). Vitamin C level of additions will give effect for $\mathrm{pH}$ condition of samples. Moreover, uniformity of $\mathrm{pH}$ for sample could mask the effects of vitamin $\mathrm{C}$ addition.

Several methods have been used to the determine EGCG content in green tea, such as the HPLC-UV (Fangueiro et al., 2014) and TLC-densitometry (Abdelsalam et al., 2014). The HPLC method offer high percentage of recovery (99-104\%), however it requires solvent and mobile phases with high purity (HPLC grade), needs high cost, is complex to operate, and had slower analysis time. The TLC-densitometry can use pro analysis grade solvent and mobile phase, low cost, simple to operate, less analysis time, and environmentally friendly. Based on the explanation, TLC-densitometry was chosen to analysis of EGCG stability in green tea (Abdelsalam et al., 2014).

The aim of this study was to determine the percentage reduction of EGCG before and after vitamin $\mathrm{C}$ addition.

\section{MATERIALS AND METHODS}

\section{Materials}

\section{Chemicals analysis}

EGCG standard was purchased from Sigma (Sigma Chemical Co., St. Louis, MO, USA). The solvent for extraction and for mobile phases were chloroform pro analysis (Merck), ethyl acetate pro analysis (Fulltime), formic acid pro analysis (Merck), acetone pro analysis (Smart-Lab). Vitamin C was obtained from Sigma Chemical, and methanol pro analysis (Fulltime) and the stationary phase of TLC silica gel aluminum plate 60F-254 (Merck made in Germany) were used. Some chemical materials for determination of vitamin $\mathrm{C}$, concentration such as potasium iodate solution $0.002 \mathrm{~mol} \mathrm{~L}-1$, starch indicator solution $0.5 \%$, potassium iodide solution 0.6 mol L-1 and hydrochloric acid 1 mol L-1 were also used.

\section{Green tea material}

A commercial green tea powdered used in this research was Meditea ${ }^{\circledR}$ (food grade) was purchased in Surabaya, Indonesia.

\section{Instruments}

Microanalytical balance (Mattler Toledo XPE26), analytic scale balance (Ohaus PA-214), ultrasonic bath (Branson 3510), Burrette, Chromatography chamber (Camag, 20 x $10 \mathrm{~cm}$ ), pH Meter (Ohaus starter 3000), TLC Plate (TLC Silica gel 60 F254, Merck), Camag Linomat 5, Camag TLC Scanner 4, Camag TLC Visualizer 2.

\section{Methods}

\section{Vitamin C standard solution}

Concentration of 500 ppm of vitamin C solution was freshly prepared from $12.5 \mathrm{mg}$ of vitamin C standard in $25 \mathrm{~mL}$ of aqueous solution.

\section{Green tea solution preparation}

Distilled water was firstly heated at $80^{\circ} \mathrm{C}$. As much as $0.25 \mathrm{~g}$ of tea powder in six replicates were immersed for 15 minutes in $15 \mathrm{~mL}$ of distilled water (in $25 \mathrm{~mL}$ volumetric flask) in ultrasonic bath. After 10 minutes, 2, 3, 4, 5, and $6 \mathrm{~mL}$ of vitamin $\mathrm{C}$ (500 ppm) were added to the sample solution and followed by distilled water addition up to the required volume. Then, the sample solutions were kept in dark glass bottle at $4^{\circ} \mathrm{C}$ (Das \& Eun, 2018).

\section{Extraction of green tea solution}

Three $\mathrm{mL}$ of green tea infusion was extracted twice with $3 \mathrm{~mL}$ of chloroform. The remaining aqueous layer was extracted twice with $3 \mathrm{~mL}$ of ethyl acetate. The ethyl acetate layer containing EGCG was then collected and evaporated in a fume hood.

\section{EGCG standard solution}

Pure standard of (-)-epigallocatechin gallate (EGCG) was prepared in methanol. Six concentrations of the calibration solution were made at the level of 50, 100, 200, 300, 400, and 500 ppm.

\section{Maximum wavelength optimization of EGCG}

The maximum wavelength of EGCG was measured in EGCG solutions (100 and 200 ppm). The wavelength which showed the highest absorbance was chosen for the next analysis.

\section{TLC condition}

The chamber was previously saturated for 2 hours with chloroform:acetone:Acetic acid (10:8:1) as a mobile phase. Densitometric scanning was performed with TLC scanner at $\lambda 278 \mathrm{~nm}$. The slit dimension was 
$4.00 \times 0.30 \mathrm{~mm}$. The scanning speed was $20 \mathrm{~mm} / \mathrm{s}$. The plate was activated in an oven at $100^{\circ} \mathrm{C}$ for 15 minutes.

\section{Vitamin C determination}

As much as $10 \mathrm{~mL}$ of GT, GTVC1, GTVC2, GTVC3, GTVC4 and GTVC5 was taken and moved into $250 \mathrm{~mL}$ of conical flash and $75 \mathrm{~mL}$ of distilated water, $2.5 \mathrm{~mL}$ of potassium iodide solution, $2.5 \mathrm{~mL}$ of hydrochloric acid solution, and $0.5 \mathrm{~mL}$ of starch indicator were added. And then, the sample was titrated with potassium iodate solution until the endpoint. The endpoint was achieved when the color of sample changes to dark-blue. Note the volume of potassium iodate needed.

\section{Validation}

Selectivity

The selectivity test was implemented by determining the separation peak of EGCG and the closest peak to it. EGCG solution (500 ppm) and extract solution were prepared. All of the samples were filtered using a membrane filter $(0.45 \mu \mathrm{m})$. The EGCG standard and extract solutions were spotted to the TLC plate, eluted, and scanned using a densitometer. The selectivity test parameters were a resolution (Rs), Rf, and peak purity. The acceptance of resolution value for Rs is $\geqslant 1.25$, Rf between $0.3-0.8$, and peak purity value close to 1.0 .

\section{Linearity}

The linearity test was analyzed by preparing 10 different concentration solutions of standard (500, 450, 400, 350, 300, 250, 200, 150, 100 and 50 ppm). All EGCG standard solutions were spotted to the TLC plates, eluted and scanned using a densitometer. A regression line was made from the concentration of EGCG standard (x) and area (y).

\section{Accuracy}

The accuracy test was analyzed by spiking three different concentrations $(80 \%, 100 \%$, and $120 \%)$ were made to sample. Three replicates were made for every concentration of EGCG standard addition. All of the samples were filtered using a membrane filter $(0.45$ $\mu \mathrm{m})$. Then, all samples and EGCG standards were spotted to the TLC plate, eluted, and scanned using a densitometer. The sample solution without standard addition was also analyzed. The quantity of determined EGCG was determinate from the corresponding calibration curve. The accuracy requirement is assessed based on the recovery. The recovery for $1-10 \%$ of EGCG in the sample was between $92-105 \%$ (AOAC International, 2013).

\section{Precision}

The precision test was analyzed by spiking a $100 \%$ concentration solution of EGCG standard to sample solution (six replications). All of the samples and EGCG standard were filtered using membrane filter $(0.45 \mu \mathrm{m})$. Then, all samples were spotted to the TLC plate, eluted, and scanned using a densitometer. The sample solution without standard addition was also analyzed. EGCG was determinated from the corresponding calibration curve. The precision requirement is assessed the RSD value. The RSD for $1-10 \%$ of EGCG in the sample is $<2.7 \%$ (AOAC International, 2013).

\section{EGCG determination}

EGCG determination was analyzed on green tea solution without vitamin $\mathrm{C}$ addition (GT), green tea with $1 \mathrm{mg}$ of vitamin addition (GTVC1), green tea with $1.5 \mathrm{mg}$ of vitamin addition (GTVC2), green tea with $2 \mathrm{mg}$ of vitamin addition (GTVC3), green tea with $2.5 \mathrm{mg}$ of vitamin addition (GTVC4) and green tea with $3 \mathrm{mg}$ of vitamin addition (GTVC5). Evaluations of EGCG were conducted at 0 day, 1 day, 2 days, 3 days, and 4 days of storage time. All extracts were dissolved in $2 \mathrm{~mL}$ of methanol p.a and filtered using membrane filter $(0.45 \mu \mathrm{m})$. All samples were spotted to the TLC plate, eluted and scanned using a densitometer. The EGCG quantity was determined from the corresponding calibration curve.

\section{Statistical analysis}

Results of EGCG values were expressed as the mean value \pm standard deviation of three independent experiments $(n=3)$. Statistical analysis was performed by one-way analysis of variance (ANOVA) using IBM SPSS statistics software version 24. A comparison of means was carried out by Tukey's multiple range analyses at $\mathrm{P}<0.05$.

\section{RESULT AND DISCUSSION \\ Extraction of green tea solution}

Chloroform, a non polar solvent, was used to remove caffeine and pigments in the green tea solution. Ethyl acetate, a semi-polar solvent, was used to extract EGCG of green tea. EGCG is a semi-polar compound so that EGCG will be extracted in the ethyl acetate layer. The ethyl acetate solution was evaporated at room temperature because of the boiling point of ethyl acetate is smaller than EGCG, ethyl acetate will evaporate but not for EGCG. And then, dry extract was dissolved with methanol. Methanol gives better separation and more volatile than ethyl acetate. 


\section{Wavelength optimization}

The maximum wavelength in $100 \mathrm{ppm}$ and 200 ppm of EGCG standard solution were $278 \mathrm{~nm}$. This maximum wavelength would be used in a densitometer scanner.

\section{Mobile optimization}

Mobile phase with different ratios were tried, such as chloroform:acetone:formic acid (10:8:3 v/v/v), chloroform:acetone:formic acid (10:8:2 v/v/v) and chloroform:acetone:formic acid (10:8:1 v/v/v). The results ware evaluated with respect to the efficiency of separation ( $\mathrm{Rf}$ and $\mathrm{Rs}$ ). The optimum mobile phase ratio was found to be chloroform:acetone:formic acid $(10: 8: 1 \mathrm{v} / \mathrm{v} / \mathrm{v})$ because the Rf of EGCG was 0.49 (acceptable value $\mathrm{Rf}=0.3-0.8$ ) and the resolution (Rs) was 1.27 (acceptable value Rs > 1.25). The densitogram of samples with different mobile phase can be seen in Table 1 .

Table 1. EGCG content in various samples

\begin{tabular}{ccccccc}
\multirow{2}{*}{ Time storage } & \multicolumn{7}{c}{ EGCG Content \pm SD $(\%$ w/w $)$} \\
\cline { 2 - 7 } & GT & GTVC1 & GTVC2 & GTVC3 & GTVC4 & GTVC5 \\
\hline 0 day & $2.568 \pm 0.81$ & $2.384 \pm 0.16$ & $2.389 \pm 0.08$ & $2.62 \pm 0.35$ & $3.027 \pm 0.87$ & $2.346 \pm 0.225$ \\
\hline 1 day & $2.151 \pm 0.31$ & $2.022 \pm 0.03$ & $1.667 \pm 0.38$ & $2.017 \pm 0.29$ & $1.903 \pm 0.22$ & $2.163 \pm 0.06$ \\
\hline 2 days & $2.125 \pm 0.06$ & $2.081 \pm 0.16$ & $2.060 \pm 0.025$ & $1.967 \pm 0.026$ & $2.061 \pm 0.14$ & $2.172 \pm 0.15$ \\
\hline 3 days & $2.059 \pm 0.15$ & $2.247 \pm 0.19$ & $2.409 \pm 0.16$ & $1.995 \pm 0.31$ & $1.699 \pm 0.42$ & $2.142 \pm 0.05$ \\
\hline 4 days & $2.056 \pm 0.21$ & $2.125 \pm 0.02$ & $1.8856 \pm 0.02$ & $2.145 \pm 0.09$ & $1.163 \pm 0.05$ & $2.117 \pm 0.04$
\end{tabular}

Noted: GT: green tea, GTVC1: green tea with $1 \mathrm{mg}$ of vitamin C addition, GTVC2: green tea with $1.5 \mathrm{mg}$ of vitamin C addition, GTVC3: green tea with $2 \mathrm{mg}$ of vitamin C addition, GTVC4: green tea with $2.5 \mathrm{mg}$ of vitamin $\mathrm{C}$ addition, GTVC5: green tea with $3 \mathrm{mg}$ of vitamin $\mathrm{C}$ addition

\section{Validation}

\section{Selectivity}

Acceptance criteria for selectivity tests are a resolution (Rs), Rf, and peak purity. The densitograms of all the samples can be seen in Figure 1. The Rs value of EGCG standard peak was 2.95 and the average Rs value of EGCG peak in the samples was 1.27. The
EGCG peak of standard and in the sample have the same Rf value (0.41). The purity of the EGCG peak standard and sample is more than 0.99. Based on the value of parameters, methods which was used for this research was selective to analyze EGCG in green tea solution.

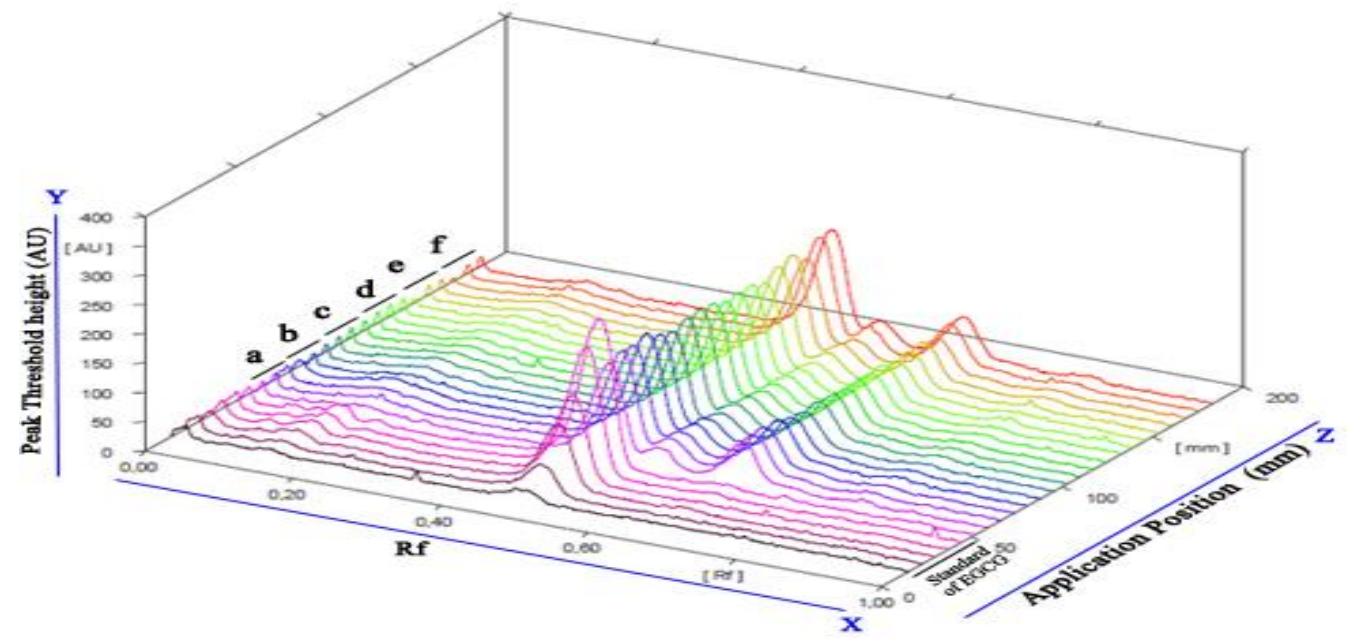

Figure 1. Densitogram of scanned TLC plate from green tea without vitamin C addition (a), green tea with $1 \mathrm{mg}$ of vitamin $\mathrm{C}$ addition (b), green tea with $1.5 \mathrm{mg}$ of vitamin $\mathrm{C}$ addition (c), green tea with $2 \mathrm{mg}$ of vitamin $\mathrm{C}$ addition (d), green tea with $2.5 \mathrm{mg}$ of vitamin $\mathrm{C}$ addition (e) and green tea with $3 \mathrm{mg}$ of vitamin $\mathrm{C}$ addition (f)

\section{Linearity}

The linearity test was carried out in a range of EGCG concentration of $0.10276 \mu \mathrm{g} / \mathrm{spot}$ up to
$1.0276 \mu \mathrm{g} / \mathrm{spot}$. This research evaluates the linearity using correlation coefficient ( $\mathrm{r}$ ) with acceptance criteria $\mathrm{r} \sim 1$, relative process standard deviation value $(\mathrm{Vxo})$ 
with acceptance criteria $\mathrm{Vxo} \leq 5 \%$ and ANOVA linearity testing with acceptance criteria sig $\leq 0.05$. The result of the correlation coefficient (r) was obtained $\mathrm{r}=0.996$ which resulted in a regression line equation $\mathrm{y}=7363 \mathrm{x}+622.5$. The relative process standard deviation value (Vxo) was obtained $3.48 \%$, and ANOVA linearity testing was obtained sig $=0.00$. So, it could be concluded that the data was linear.

\section{Accuracy}

Accuracy test was analyzed by spiking $80 \%, 100 \%$ and $120 \%$ of EGCG standard to the sample and measure the recovery. Acceptance criteria of recovery for $1 \%-10 \%$ analyte concentration is $92 \%-105 \%$ (AOAC International, 2013). The recovery of $80 \%$, $100 \%$, and $120 \%$ of EGCG standard addition were $99.330 \% \pm 1.52,103.753 \% \pm 1.04$, and $93.632 \% \pm$ 0.37 respectively. The results indicated that this method was accurate.

\section{Precision}

The precision test was analyzed by spiking $100 \%$ of the EGCG standard (six replicates). The precision measure RSD value. Acceptance criteria of RSD for $1 \%-10 \%$ analyte concentration is $\leq 2.7 \%$ (AOAC International, 2013). The result of RSD was $1.006 \%$ with recovery $103.754 \pm 1.04$. The results suggested that this method was precision.

\section{Stability of EGCG value in green tea solution}

Evaluation of EGCG value in all of samples was analyzed in one TLC plate (Figure 2). EGCG value was determined from the corresponding calibration curve. The result of EGCG level in all samples which were conducted at 0 day, 1 day, 2 days, 3 days and 4 days of storage can be seen in Table 1 . The stability of EGCG was determined from the percent loss of EGCG during storage time (Table 2). Percent loss of EGCG was calculated by the formula :

$$
\begin{aligned}
& \% \text { Loss of EGCG }=\frac{\mathrm{B}_{0}-\mathrm{B}_{\mathrm{n}}}{\mathrm{B}_{0}} \times 100 \% \\
& \mathrm{Bn}=\text { EGCG value at } \mathrm{n} \text { day storage, } \mathrm{n}=1,2,3,4 \text { day } \\
& \mathrm{B} 0=\mathrm{EGCG} \text { value at } 0 \text { day storage }
\end{aligned}
$$

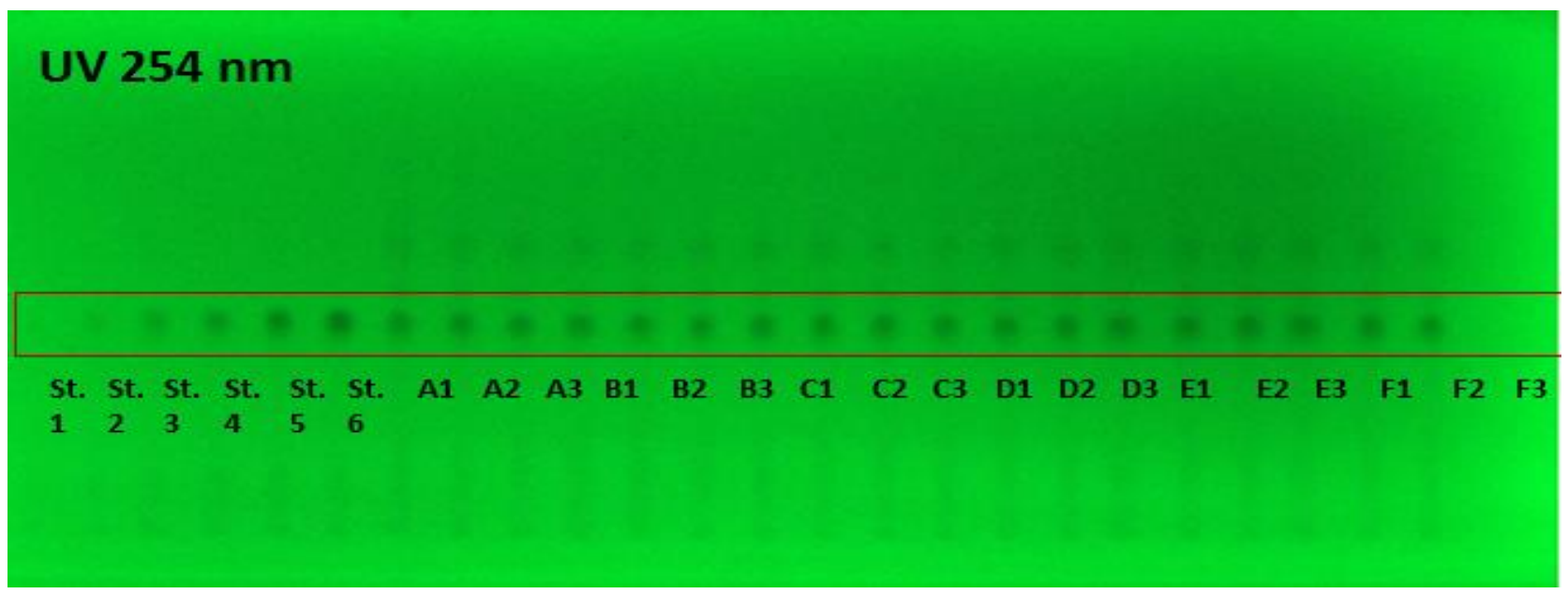

Figure 2. Visualization of TLC plate after eluted. 50 ppm - 500 ppm EGCG standard (St1-St6); green tea extract (A1A3); green tea + vitamin C $1 \mathrm{mg}$ extract (B1-B3); green tea + vitamin C $1.5 \mathrm{mg}$ extract C $1.5 \mathrm{mg}(\mathrm{C} 1-\mathrm{C} 3)$; green tea + vitamin C $2 \mathrm{mg}$ extract (D1-D3); green tea + vitamin C $2.5 \mathrm{mg}$ extract (E1-E3) and green tea + vitamin C3 mg extract (F1-F3)

Table 2. \% Degreasing of EGCG content in all sample

\begin{tabular}{ccccccc}
\hline \multirow{2}{*}{$\begin{array}{c}\text { Time } \\
\text { storage }\end{array}$} & GT & GTVC1 & GTVC2 & GTVC3 & GTVC4 & GTVC5 \\
\cline { 2 - 7 } 1 day & $16.23 \pm 0.31$ & $15.18 \pm 0.33$ & $30.23 \pm 0.48$ & $23.05 \pm 1,35$ & $28.56 \pm 0.82$ & $7.78 \pm 0.96$ \\
\hline 2 days & $17.26 \pm 0.86$ & $12.74 \pm 0.96$ & $13.76 \pm 0.85$ & $24.95 \pm 0,69$ & $31.93 \pm 0.94$ & $7.41 \pm 0.55$ \\
\hline 3 days & $19.85 \pm 0.95$ & $5.74 \pm 0.59$ & $-0.87 \pm 0.96^{*}$ & $23.88 \pm 0,86$ & $43.89 \pm 0.52$ & $8.71 \pm .85$ \\
\hline 4 days & $19.93 \pm 0.91$ & $10.89 \pm 0.92$ & $21.08 \pm 0.92$ & $18.18 \pm 0,21$ & $28.56 \pm 1.05$ & $9.76 \pm 1.04$ \\
\hline
\end{tabular}

${ }^{*}=$ increasing of EGCG content

GT: green tea, GTVC1: green tea with $1 \mathrm{mg}$ of vitamin C addition, GTVC2: green tea with $1.5 \mathrm{mg}$ of vitamin C addition, GTVC3: green tea with $2 \mathrm{mg}$ of vitamin C addition, GTVC4: green tea with $2.5 \mathrm{mg}$ of vitamin C addition, GTVC5: green tea with $3 \mathrm{mg}$ of vitamin $\mathrm{C}$ addition 
The percent loss of EGCG in GT, GTVC1, GTVC2, GTVC3, GTVC4 and GTVC5 after 4 days storage were $19.93 \%, 10.89 \%, 21.08 \%, 18.18 \%$, $28.56 \%$, and $9.76 \%$, respectively. There were statistically significant differences $(\mathrm{p}<0.05)$ of percent loss of EGCG between samples.

Significant differences value show that vitamin C addition affected to \% loss of EGCG. Three mg of vitamin $\mathrm{C}$ addition (GTVC5) was the best result which prevents decreasing of EGCG in green tea solution. GTVC5 has \% loss of EGCG less then other samples.

Vitamin C can stabilize EGCG in green tea solution. The potential reductions of vitamin $\mathrm{C}$ and EGCG are $282 \mathrm{mV}$ and $430 \mathrm{mV}$ respectively. EGCG potential reduction is greater than vitamin $\mathrm{C}$. It caused that EGCG is easier to attract electrons and vitamin C is easier to release electrons (Tsao, 2015). EGCG will be degraded to GCG or gallocatechin gallate and theasinensin A. Vitamin $\mathrm{C}$ in green tea solution is easily oxidized from diketo form to as dehydroascorbic acid, which followed by conversion into oxalic acid and threonic acid (Lung \& Destiani, 2014). The EGCG degradation will attract electrons from vitamin $\mathrm{C}$ easily, so EGCG radical regenerated to normal EGCG. The prediction of stability reaction between EGCG and vitamin $\mathrm{C}$ can be seen in Figure 3. Oxidation of vitamin $\mathrm{C}$ forms oxalic and trienoic acid (Truffault $e t$ al., 2017). This was proofed by decreasing vitamin $C$ levels in green tea solution during 4 days storage. The average of decreased level of vitamin $\mathrm{C}$ in GT, GTVC1, GTVC2, GTVC3, GTVC4 and GTVC5 were $36.7 \%, 38.4 \%, 41.8 \%, 42.1 \%, 42.9 \%$, and $47.5 \%$, respectively.

Electron from the degradation of vitamin $\mathrm{C}$ will regenerate radical EGCG into non-radical EGCG (Dai et al., 2008). This theory was in line with the result of this research. The more vitamin $\mathrm{C}$ levels decreased, the more EGCG radicals generated to be EGCG.

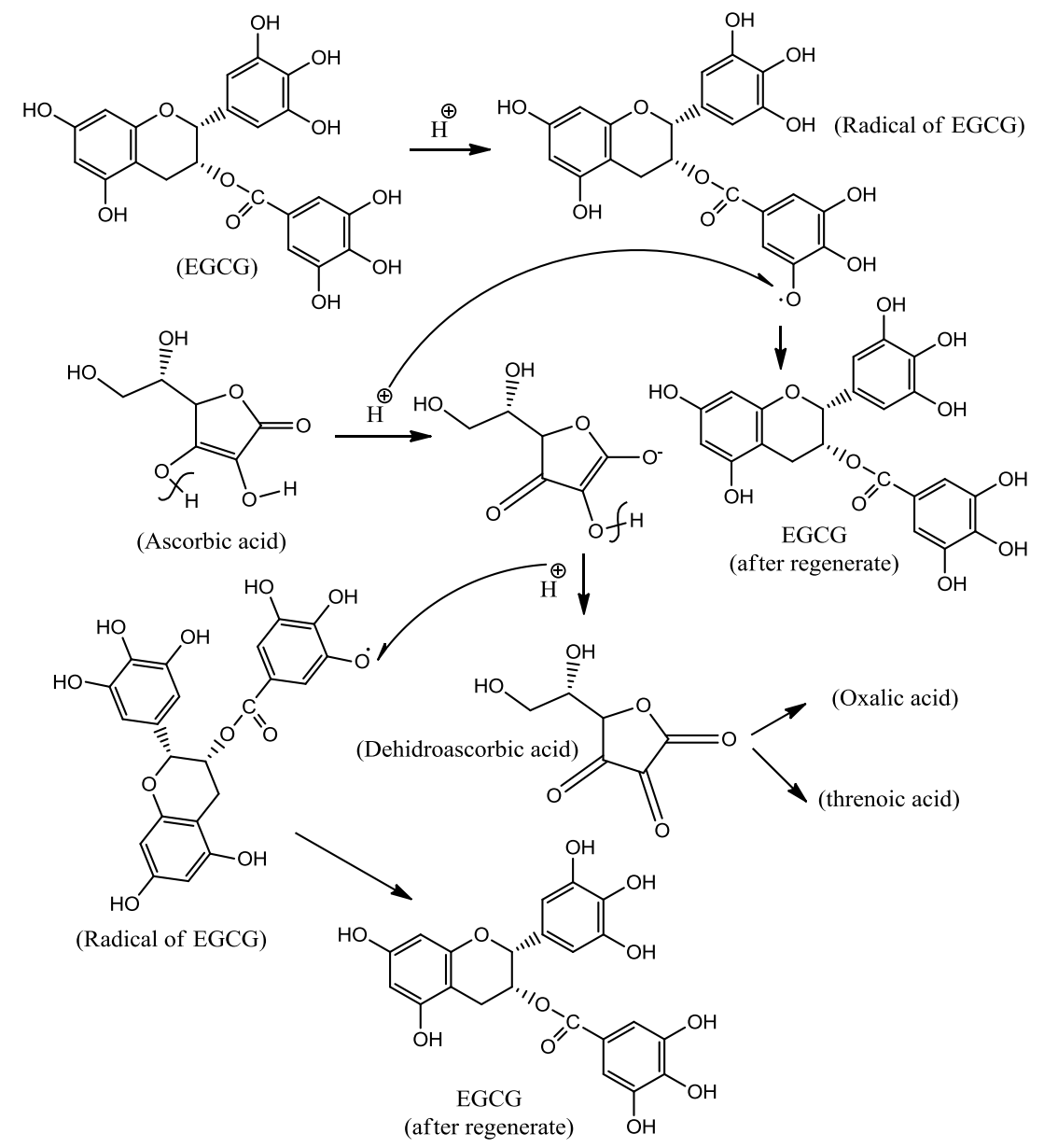

Figure 3. Prediction of EGCG stability reaction by vitamin C

\section{CONCLUSION}

The result of this study showed that during 4 days of storage of EGCG revealed that a precentace reduction of EGCG in green tea solution with $3 \mathrm{mg}$ of vitamin C addition (GTVC5) was 9.76\%. It was smaller than green tea solution without vitamin C addition (GT) which decreased $19.93 \%$ of EGCG. 


\section{REFERENCES}

Abdelsalam, H. H., Al-ghobashy, M. A., Zaazaa, H. E. \& Ibrahim, M. A. (2014). Stability of Catechins in Green Tea Nutraceutical Products: Application of Solid Phase Extraction - Thin Layer Chromatography Densitometry. Food Chemistry; 156; 94-99.

AOAC International. (2013). AOAC Official Methods of Analysis - Appendix K: Guidelines for Dietary Supplements and Botanicals. Rockville: AOAC International.

Dai, F., Chen, W. \& Zhou, B. (2008). Antioxidant Synergism of Green Tea Polyphenols with ATocopherol and L-Ascorbic Acid in SDS Micelles. Biochimie; 90; 1499-1505.

Das, P. R. \& Eun, J. B. (2018). A Comparative Study of Ultra-sonication and Agitation Extraction Techniques on Bioactive Metabolites of Green Tea Extract. Food Chemistry; 253; 22-29.

Fangueiro, J. F., Parra, A., Silva, A. M., Egea, M. A., Souto, E. B., Garcia, M. L. \& Calpena, A. C. (2014). Validation of a High Performance Liquid Chromatography Method for the Stabilization of Epigallocatechin Gallate. International Journal of Pharmaceutics; 475; 181-190.

Kosugi, Y. Green Tea Consumption. http://www.ocha.net/english/teacha/distribution/greentea3.ht ml. Accessed: 25 January 2019.
Krupkova, O., Ferguson, S. J. \& Wuertz-Kozak, K. (2016). Stability of (-)-Epigallocatechin Gallate and Its Activity in Liquid Formulations and Delivery Systems. Journal of Nutritional Biochemistry; 37; 1-12.

Lung, J. K. S. \& Destiani, D. P. (2014). Uji Aktivitas Antioksidan Vitamin A, C, E dengan Metode DPPH. Farmaka; 14; 1-10.

Rady, I., Mohamed, H., Rady, M., Siddiqui, I. A. \& Mukhtar, H. (2018). Cancer Preventive and Therapeutic Effects of EGCG, the Major Polyphenol in Green Tea. Egyptian Journal of Basic and Applied Sciences; 5; 1-23.

Truffault, V., Fry, S. C., Stevens, R. G. \& Gautier, H. (2017). Ascorbate Degradation in Tomato Leads to Accumulation of Oxalate, Threonate and Oxalyl Threonate. Plant Journal; 89; 996-1008.

Tsao, R. (2015). Synergistic Interactions Between Antioxidants Used in Food Preservation. In: Handbook of Antioxidants for Food Preservation. Sawston: Woodhead Publishing.

Wu, J., Chiang, M., Chang, Y., Chen, J., Yang, H., Lii, C. \& Yao, H. (2011). Correlation of Major Components and Radical Scavenging Activity of Commercial Tea Drinks in Taiwan. Journal of Food and Drug Analysis; 19; 289-300.

Zeng, L., Ma, M., Li, C. \& Luo, L. (2017). Stability of Tea Polyphenols Solution with Different $\mathrm{pH}$ at Different Temperatures. International Journal of Food Properties; 20; 1-18. 